\title{
Intoxicaciones agudas en pacientes mayores de 65 años
}

\section{Acute poisoning in patients over 65 years of age}

\author{
P. Miranda Arto' , A. Ferrer Dufol ${ }^{2}$, F.J. Ruiz Ruiz ${ }^{3}$, S. Menao Guillén², E. Civeira Murillo ${ }^{4}$
}

\section{RESUMEN}

Fundamento. Los estudios sobre la intoxicación aguda en sujetos de edad avanzada en nuestro país son escasos, a pesar de los riesgos asociados que presenta este grupo de pacientes.

Material y métodos. Estudio descriptivo retrospectivo de las intoxicaciones agudas atendidas en el servicio de Urgencias del Hospital Clínico Universitario de Zaragoza entre 1995 y 2009 , en pacientes con edad igual o mayor a 65 años.

Resultados. Se registraron 762 casos, el 4,74\% del total de intoxicados, con una edad media de $74,16(\mathrm{SD} \pm 6)$ años. La vía oral fue la más frecuente (85\%) y por tipo de intoxicación, la sobredosis de alcohol (28,7\%). Junto a éste, las benzodiacepinas (14,3\%) y los productos cáusticos (11\%) fueron los principales tóxicos involucrados. El $21 \%$ de los pacientes recibieron tratamiento específico antitóxico, no precisando ingreso el $82,4 \%$. Se observó en la evolución temporal una tendencia que apuntaba a la disminución del tratamiento emético y del lavado gástrico y un aumento del carbón activado. La mortalidad de la serie fue del $1,04 \%$.

Conclusiones. Las intoxicaciones en pacientes mayores presentan una mayor morbimortalidad, precisando más ingresos y más tentativas autolíticas con resultado de muerte.

Palabras clave. Intoxicación. Anciano. Urgencias.

\begin{abstract}
Background. There are few Spanish studies on acute poisoning in the elderly despite the associated risk factors of this group of patients.
\end{abstract}

Material and methods. Retrospective descriptive study of acute poisonings treated in the Emergency Service of the University Hospital of Zaragoza from 1995 to 2009 on patients 65 years old or older

Results. A total of 762 patients were selected in the study (4.74\% of all acute poisonings) with a mean age of $74.16(\mathrm{SD} \pm 6)$ years. Ingestion was the major route of exposure $(85 \%)$ and alcohol overdose $(28,7 \%)$ was the most frequent type of poisoning. A trend was also observed showing a lower emetic treatment and gastric lavage and an increase in activated charcoal. Benzodiazepines (14.3\%) and toxic household products (11\%) with caustic properties were also the main toxics found in the study.

Conclusions. Acute poisonings in the elderly required more hospitalizations, have a higher mortality and more autolytic attempts which result in death.

Key words. Poisoning. Elderly. Emergency service.
1. Servicio Aragonés de Salud. Atención Primaria Sector III. Zaragoza.

2. Hospital Clínico Universitario Lozano Blesa, Unidad Toxicología Clínica. Zaragoza.

3. Hospital Clínico Universitario Lozano Blesa, Área de Urgencias. Zaragoza.

4. Hospital Clínico Universitario Lozano Blesa, Unidad de Cuidados Intensivos. Zaragoza.

\section{Correspondencia: \\ Pilar Miranda Arto}

Servicio Aragonés de Salud

Atención Primaria Sector III

Zaragoza.

pilimira@gmail.com

Recepción: 29 de octubre de 2013

Aceptación provisional: 25 de noviembre de 2013

Aceptación definitiva: 10 de febrero de 2014 


\section{INTRODUCCIÓN}

Las intoxicaciones agudas (IA) constituyen un problema de salud potencialmente grave y son motivo de consulta habitual en los servicios de urgencias hospitalarios (SUH). Durante las últimas décadas se ha observado un incremento en el número de casos, llegando a constituir el 1-2\% de las urgencias médicas atendidas en los hospitales de nuestro país ${ }^{1,2}$.

En el paciente de edad avanzada las IA se asocian a un mayor riesgo por la presencia de factores de comorbilidad y modificaciones en la toxicodinámica. Cambios en la toxicocinética, como la disminución de la capacidad de metabolización y de eliminación, producidos por el proceso de envejecimiento hepático y renal, pueden desencadenar un aumento de la toxicidad ${ }^{3}$ al verse incrementada la vida media de eliminación del tóxico. Los adultos mayores suelen tener más trastornos del ánimo y mayor riesgo de suicidio por causas económicas ${ }^{4}$, duelo, enfermedad física ${ }^{5,6}$, trastornos cognitivos, dependencia y aislamiento social, factores que a su vez, pueden producir que el paciente acuda de forma tardía al médico o a un servicio de urgencias ${ }^{7}$, implicando una mayor gravedad de la intoxicación ${ }^{8-10}$. También hay que mencionar como factores de riesgo la automedicación ${ }^{11}$, el exceso de prescripción en numerosas ocasiones ${ }^{12}$ y la "polimedicación"13, común en este tipo de pacientes debido a la pluripatología ${ }^{14,15}$.

En la bibliografía aparecen varios estudios epidemiológicos sobre la intoxicaciones agudas atendidas en los servicios de Urgencias Hospitalarios ${ }^{1,2},{ }^{16-24}$, pero es escasa la información que aportan acerca de la IA en los pacientes de mayor edad, por lo que nos planteamos como objetivo en este estudio analizar en detalle la epidemiología de las intoxicaciones atendidas en un servicio de urgencias en pacientes mayores de 65 años y sus particularidades en comparación con las intoxicaciones en la población general.

\section{MATERIAL Y MÉTODOS}

Estudio descriptivo, retrospectivo de las intoxicaciones agudas atendidas en el servicio de Urgencias del Hospital Clínico Universitario de Zaragoza, hospital de tercer nivel que atiende a una población de referencia de 300.000 personas, y que cuenta desde 1980 con una de las ocho Unidades de Toxicología Clínica (UTC) que hay en nuestro país. Dicha UTC dispone de una base de datos obtenida de forma prospectiva mediante la revisión de las historias clínicas y el posterior registro de los pacientes atendidos por sospecha clínica de exposición a un producto tóxico en el servicio de Urgencias y en la Unidad de Cuidados Intensivos del hospital.

Además de los datos epidemiológicos y clínicos, la intencionalidad de la intoxicación se clasificó en: sobredosis para casos asociados al consumo de sustancias de abuso en contexto recreativo, suicida para casos producidos por un gesto autoagresivo y accidental en los producidos de forma involuntaria (yatrogenia, domestico, laboral etc.). El agente tóxico se clasificó en: medicamento para cualquier sustancia comercializada con fines terapéuticos, drogas de abuso para sustancias adictivas empleadas usualmente con fines recreativos y otros tóxicos en el que se engloban sustancias de uso doméstico, industrial, setas, picaduras etc. Una vez analizadas las muestras remitidas a la UTC se estableció la concordancia entre los datos recogidos en la historia clínica del paciente y el resultado analítico, estableciéndose dicha concordancia cuando los datos recogidos en la anamnesis o la sospecha clínica inicial coincidían con el resultado obtenido.

Se seleccionaron los registros del periodo comprendido entre enero de 1995 y diciembre de 2009 (15 años), incluyendo como casos los pacientes con edad igual o mayor a 65 años. Los resultados se presentan con media y desviación estándar para las variables cuantitativas y porcentajes para las variables cualitativas. La normalidad de las variables se comprobó con los test de Shapiro-Wilks y Kolmogorov-Smirnov. Se emplearon los tests de Chi cuadrado y el test exacto de Fisher para analizar la asociación entre variables cualitativas y los test de t-Student o U de Mann-Whitney para las cuantitativas, según criterios de 
normalidad. Se utilizó un modelo de regresión lineal simple y se calculó $\mathrm{R}^{2}$ en el ajuste de la línea de puntos para determinar la tendencia en la evolución de los tratamientos aplicados. La significación estadística se consideró para valores de $\mathrm{p}<0,05$.

\section{RESULTADOS}

De los 16.075 pacientes del registro, 762 pacientes $(4,74 \%)$, cumplían el criterio de inclusión. La edad media y desviación estándar de la serie fue de $74,16 \pm 6$ años y el porcentaje de varones del $57,5 \%$. Hubo diferencias estadísticamente significativas en la media de edad según el sexo, con $73,06 \pm 6$ años para los hombres y 75,6 \pm 7 años para las mujeres $(\mathrm{p}<0,05)$.

El tipo de intoxicación, porcentaje y distribución por sexo comparado con el grupo de menores de 65 años, se muestran en la figura 1.

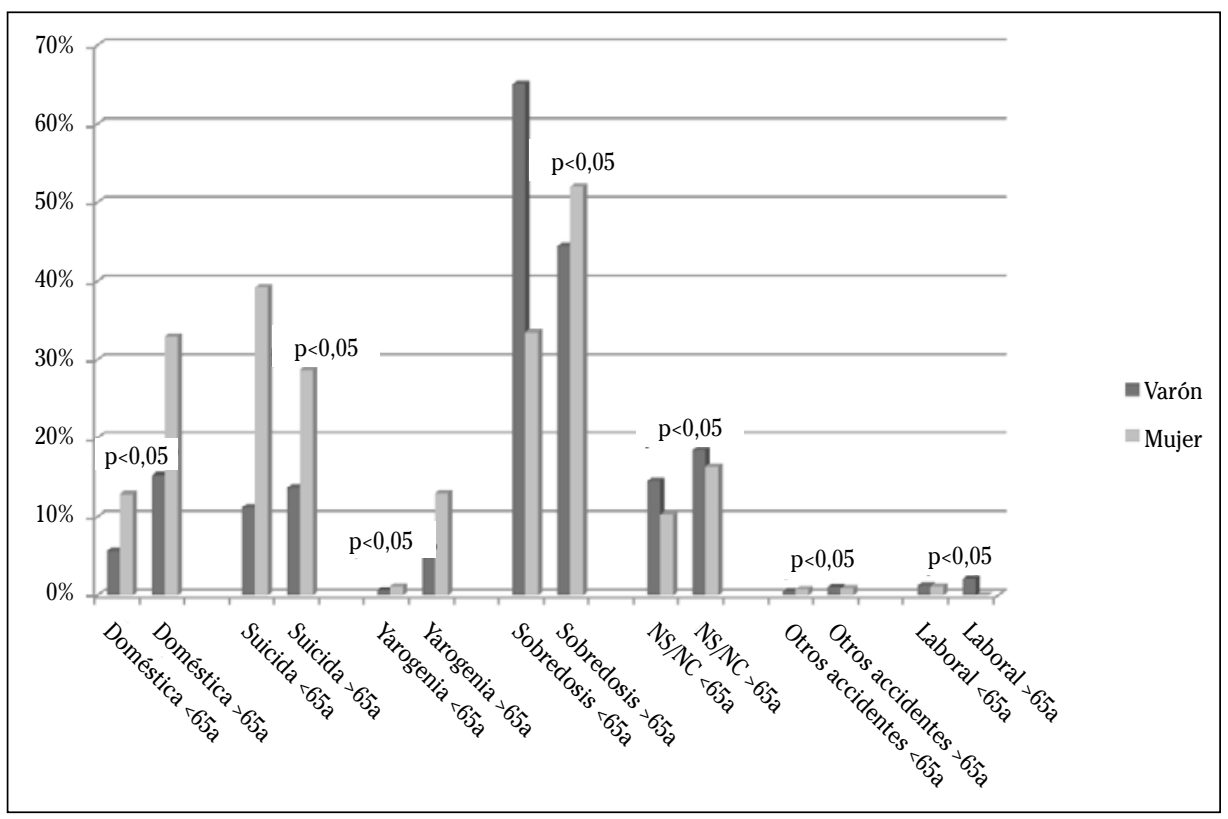

Figura 1. Porcentajes del tipo de intoxicación por sexo en mayores y menores de 65 años.

Se observó un aumento progresivo de casos desde el inicio: 25 casos anuales en 1995 (3,3\% del total de intoxicados), hasta triplicarse con un pico de 80 casos anuales (10,5\%) en los años 2005-2006 (Fig. 2).

Los fármacos más frecuentemente implicados fueron las benzodiacepinas; entre las drogas destacó el alcohol y de otros tóxicos los productos domésticos con propiedades cáusticas (Tabla 1). Analizando específicamente las intoxicaciones que se realizaron con fines autolíticos se observó un predominio ( $69,3 \%$ vs $44,4 \%$ ) de benzodiacepinas en los menores de 65 años y un predominio de cáusticos (14,3\% vs 3,6\%) y plaguicidas $(5,8 \%$ vs $1,7 \%)$ entre los mayores de 65 años $(\mathrm{p}<0,05)$.

En el 71,5\% de los casos, la IA se debió a un único tóxico, en el 8,26\% a la asociación de dos o más fármacos, y en el 1,8\% por combinación de fármaco y droga. La vía de entrada más frecuente del tóxico fue la oral en un $85 \%$, seguida de la respiratoria (12,6\%), la cutánea $(1,9 \%)$ y la parenteral $(0,5 \%)$. 


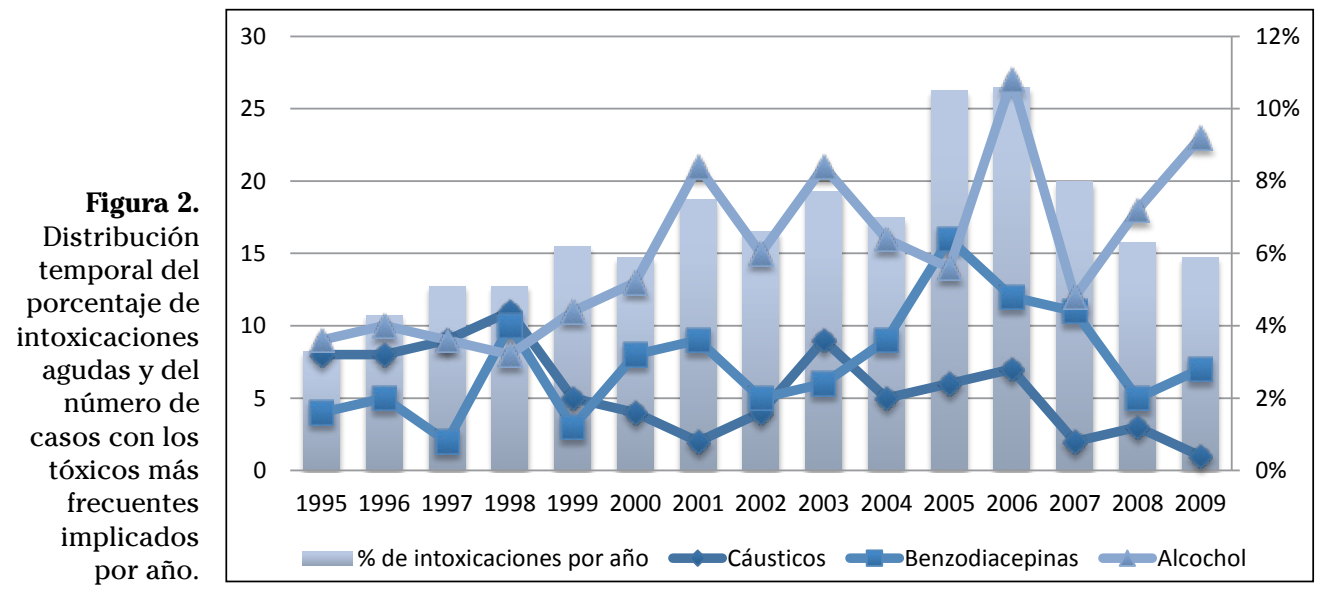

Tabla 1. Tóxicos implicados

\begin{tabular}{|c|c|c|c|}
\hline Tóxicos implicados & $\begin{array}{c}\text { Mayores de } 65 \text { años } \\
(n=762)\end{array}$ & $\begin{array}{c}\text { Menores de } 65 \text { años } \\
(n=15.293)\end{array}$ & $\mathbf{P}$ \\
\hline \multicolumn{4}{|l|}{ Medicamentos } \\
\hline Benzodiacepinas & $14,3 \%$ & $17,8 \%$ & $<0,05$ \\
\hline Antidepresivos tricíclicos & $3 \%$ & $3,2 \%$ & $\mathrm{~ns}$ \\
\hline Neurolépticos & $1,78 \%$ & $1,7 \%$ & ns \\
\hline Paracetamol & $1,6 \%$ & $1,7 \%$ & ns \\
\hline Antidepresivos ISRS & $1,57 \%$ & $1,6 \%$ & ns \\
\hline Digital & $0,5 \%$ & $0,1 \%$ & $<0,05$ \\
\hline Litio & $0,47 \%$ & $0,1 \%$ & ns \\
\hline Barbitúricos & $0,46 \%$ & $0,2 \%$ & ns \\
\hline Salicilatos & $0,38 \%$ & $0,7 \%$ & ns \\
\hline AINE & $0,35 \%$ & $1,2 \%$ & $<0,05$ \\
\hline Otros antidepresivos & $0,3 \%$ & $0,3 \%$ & ns \\
\hline Antiarrítmicos & $0,3 \%$ & $0,04 \%$ & ns \\
\hline Antiepilépticos & $0,3 \%$ & $0,21 \%$ & ns \\
\hline Otros fármacos & $12,9 \%$ & $6,6 \%$ & $<0,05$ \\
\hline \multicolumn{4}{|l|}{ Drogas } \\
\hline Alcohol & $28,7 \%$ & $50,9 \%$ & $<0,05$ \\
\hline Cocaína & $0,3 \%$ & $6,3 \%$ & $<0,05$ \\
\hline Opiáceos & $0,3 \%$ & $4,1 \%$ & $<0,05$ \\
\hline \multicolumn{4}{|l|}{ Otros tóxicos } \\
\hline Cáusticos & $11 \%$ & $2,5 \%$ & $<0,05$ \\
\hline Gases irritantes & $4,9 \%$ & $1,3 \%$ & $<0,05$ \\
\hline Monóxido de carbono & $4,6 \%$ & $1,7 \%$ & $<0,05$ \\
\hline Plaguicidas & $3,4 \%$ & $0,7 \%$ & $<0,05$ \\
\hline Setas & $1,45 \%$ & $0,4 \%$ & $<0,05$ \\
\hline Disolventes & $1,27 \%$ & $0,9 \%$ & ns \\
\hline Picaduras & $0,14 \%$ & $0,1 \%$ & ns \\
\hline Otros & $3,60 \%$ & $2 \%$ & ns \\
\hline
\end{tabular}

ns : $p>0,05$, estadísticamente no significativo 


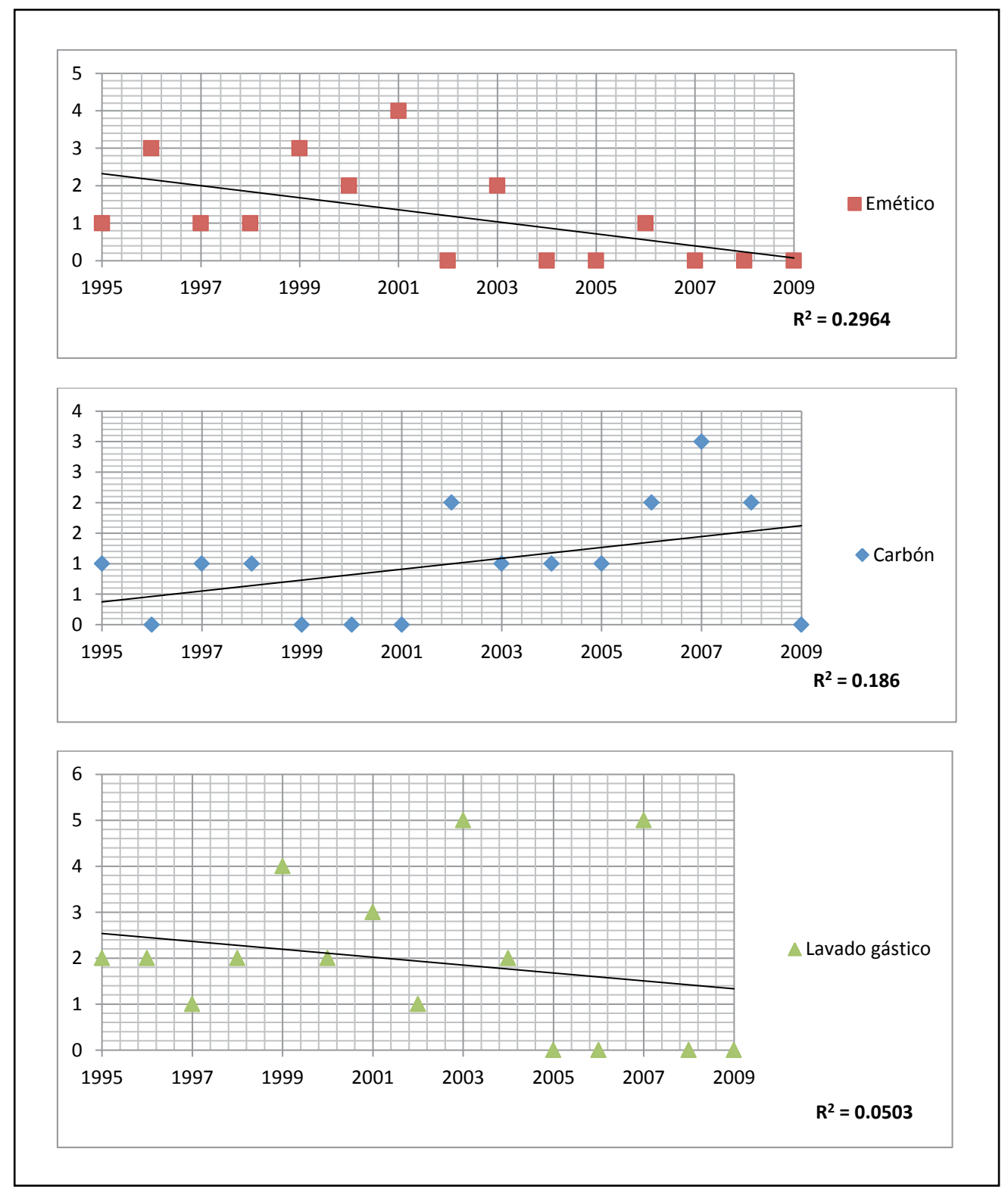

Figura 3. Distribución temporal de los tratamientos de descontaminación digestiva administrados.

En cuanto a manifestaciones clínicas, el 18\% de los pacientes presentaban síntomas de embriaguez (fetor enólico, euforia, disartria, etc). En el resto, los síntomas más frecuentes fueron la disminución del nivel de consciencia (13\%), los vómitos (9\%), la disnea $(8,1 \%)$, la taquicardia $(5,6 \%)$, el síncope $(4,5 \%)$ y la hipotensión (4\%).

Se realizó un análisis toxicológico en 426 casos $(57,3 \%)$ y sus resultados fueron concordantes con la sospecha clínica en un $52,58 \%$. 
El intervalo asistencial se registró en 64 pacientes $(8,3 \%)$. Recibieron tratamiento sintomático el $63,5 \%$ de los pacientes, y tratamiento específico el $21,2 \%$. La figura 3 muestra la evolución temporal de los tratamientos de descontaminación digestiva. El $8,8 \%$ recibió algún tratamiento de descontaminación, siendo el más frecuente el lavado gástrico (3,8\%), seguido del carbón activado (8\%). El 12,4\% de las intoxicaciones recibieron algún tratamiento antidótico, siendo el fármaco más usado el flumazenilo $(7,5 \%)$ (Tabla 2$)$. Trece pacientes $(1,7 \%)$ precisaron de intubación orotraqueal y 5 $(0,6 \%)$ la administración de drogas vasoactivas. En el 33\% de los casos atendidos se constató la existencia de antecedentes psiquiátricos. El trastorno más frecuente fue el trastorno mental y del comportamiento debido al consumo de alcohol (16,2\%) junto con el episodio depresivo $(16,2 \%)$, seguidos de trastorno bipolar $(2,8 \%)$, y esquizofrenia/trastorno esquizotípico $(1,6 \%)$. En el $2 \%$ de los casos el paciente presentaba un proceso neurodegenerativo (enfermedad de Alzheimer, demencia vascular, etc.).

Tabla 2. Tratamiento específico recibido en urgencias

\begin{tabular}{lccc}
\hline $\begin{array}{c}\text { Tratamiento } \\
\text { específico }\end{array}$ & $\begin{array}{c}\text { Mayores de 65 años } \\
(\mathbf{n = 7 6 2})\end{array}$ & $\begin{array}{c}\text { Menores de 65 años } \\
(\mathbf{n = 1 5 . 2 9 3})\end{array}$ & p \\
\hline Descontaminación & & $3,1 \%$ & $\mathrm{~ns}$ \\
\hline Lavado gástrico & $3,80 \%$ & $6 \%$ & $<0,05$ \\
\hline Emetizante & $2,40 \%$ & $1,3 \%$ & $\mathrm{~ns}$ \\
\hline Carbón activado & $1,80 \%$ & $0,5 \%$ & $\mathrm{~ns}$ \\
\hline Lavado cutáneo & $0,50 \%$ & $0,2 \%$ & $\mathrm{~ns}$ \\
\hline Lavado ocular & $0,30 \%$ & & \\
\hline Antídoto & & $5,3 \%$ & $<0,05$ \\
\hline Flumazenilo & $7,50 \%$ & $0,5 \%$ & $<0,05$ \\
\hline Oxígeno a alto flujo & $2,10 \%$ & $0 \%$ & $<0,05$ \\
\hline Atropina & $1 \%$ & $0,3 \%$ & $\mathrm{n}$ \\
\hline N-acetilcisteína & $0,70 \%$ & $0,8 \%$ & $<0,05$ \\
\hline Naloxona & $0,50 \%$ & $0,01 \%$ & $<0,05$ \\
\hline Vit K & $0,30 \%$ & $0 \%$ & \\
\hline Hidroxicobalamina & $0,10 \%$ & & \\
\hline
\end{tabular}

ns : p>0,05, estadísticamente no significativo

El destino del paciente desde el servicio de Urgencias fue el domicilio en un $82,4 \%$, bien de forma directa tras su valoración y tratamiento $(42,6 \%)$, bien tras un periodo de vigilancia en la sala de observación del servicio de Urgencias (39,8\%). El resto de los pacientes fueron ingresados en alguna de las unidades asistenciales del hospital (Fig. 4).

Se registraron 8 fallecimientos $(1,04 \%)$, de los que 5 fueron hombres $(62,5 \%)$. La causa en 7 casos fue suicida ( 3 mujeres y 4 hombres), en todos ellos por la ingesta de un cáustico. El octavo fallecimiento se debió a la ingesta accidental de un plaguicida organofosforado. Entre los mayores de 65 años se registraron 8 defunciones $(1,04 \%)$ frente a las 20 defunciones $(0,13 \%)$, registradas en el grupo de menores de 65 años, siendo la diferencia estadísticamente significativa $(\mathrm{p}<0,05)$. 


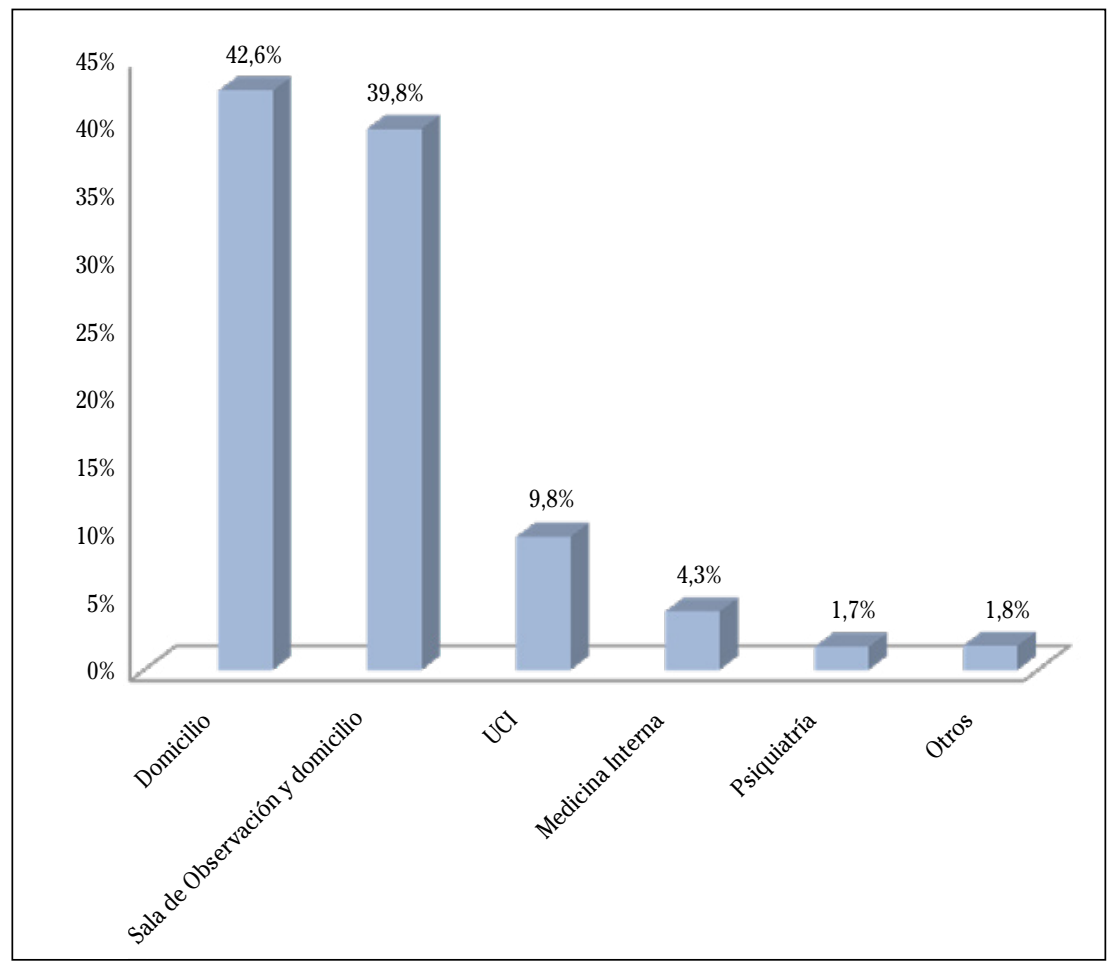

Figura 4. Destino del paciente después de su valoración en el servicio de Urgencias

\section{DISCUSIÓN}

A pesar del incremento progresivo de las consultas por intoxicación aguda en los SUH reflejado en estudios de ámbito nacional $^{1,2}$ e internacional ${ }^{3,25}$, del aumento global de las visitas a los $\mathrm{SUH}^{26}$ y del incremento del número de pacientes mayores atendidos en el servicio de Urgencias, que en el caso de nuestro hospital suponen el $25 \%$ del total de las urgencias atendidas anuales (incluida la población pediátrica) que están en cifras alrededor de las 120.000, la incidencia de intoxicación aguda en pacientes de edad avanzada en nuestro estudio es baja (4,74\% del total de intoxicaciones). Ello podría ser uno de los motivos por los que no se han desarrollado más estudios en España sobre este tema, y confirma la pertinencia de este trabajo ${ }^{26}$.
Datos obtenidos de estudios realizados en otros países reflejan mayor porcentaje de intoxicaciones en mujeres de edad avanzada, con un rango que oscila entre el $57,5 \%$ y el $61 \%$, lo que contrasta con el $42 \%$ de nuestra serie, probablemente debido al hecho de haber incluido en nuestro trabajo la intoxicación etílica. Doak y col ${ }^{3}$ argumentan que las mujeres son más propensas a intoxicaciones de tipo suicida que los hombres, aunque con una menor tasa de mortalidad, lo que coincide con nuestros resultados.

El tóxico más frecuentemente implicado en nuestro estudio fue el alcohol, aunque sin constatar muertes directamente producidas por el mismo. Aunque son conocidos los efectos nocivos de la ingesta continuada de alcohol a largo plazo, Friedmann y col ${ }^{27}$ no encontraron efectos 
sobre el estado de salud física en pacientes ancianos con problemas relacionados con la ingesta aguda de alcohol atendidos en un SUH. En otro estudio realizado en Alemania $^{28}$, se demostró que los ancianos ingresados por intoxicación en un servicio de cuidados intensivos no prolongaban su estancia en dicha unidad en el caso de la intoxicación por alcohol.

Las benzodiacepinas y antidepresivos aparecen como fármacos más frecuentes, dato que coincide con el estudio de Pichot $\mathrm{MH}$ y $\mathrm{col}^{29}$. A pesar de no ser objetivo directo de nuestra investigación, en España los eventos adversos por fármacos suponen entre un 7,2 y un $14 \%$ de los ingresos de pacientes ancianos en servicios de medicina interna ${ }^{30}$. El consumo de medicamentos durante las últimas décadas se ha incrementado desde 3 hasta 8,2 fármacos/ día y se ha demostrado que el número de fármacos ingeridos se relaciona con los efectos adversos ${ }^{31-33}$. La tasa de prescripción inadecuada en mayores de 65 años en el ámbito de la atención primaria se sitúa en torno al $20,5 \%$, con cifras que van del 14 al $37 \%{ }^{34}$. La "polimedicación", el acceso financiado o parcialmente financiado, la confusión o uso indebido, el almacenamiento inadecuado, y el deterioro cognitivo puede conducir a un aumento de la intoxicación mixta. Por ello sería recomendable tomar iniciativas para su prevención, limitando la "polifarmacia", simplificando la dosificación de la misma y revisando de forma periódica la medicación crónica prescrita. A ello habría que añadir las recomendaciones a familiares o cuidadores de las personas con deterioro cognitivo y la administración de medicación bajo supervisión directa. Todas estas medidas podrían ayudar a reducir la frecuencia y morbilidad de las intoxicaciones en personas de edad avanzada ${ }^{35}$.

Las recomendaciones de la European Association of Poison Centres and Clinical Toxicologists y de la American Academy of Clinical Toxicology ${ }^{36,37}$, desaconsejan el uso del lavado gástrico de forma rutinaria y proponen el carbón activado para tóxicos absorbibles por el mismo con un margen de una hora desde la ingesta del tóxico. En nuestro estudio se observó un uso mayor del lavado gástrico en detrimento del carbón activado. En estos datos podría influir el hecho de tratarse de una serie de 15 años, pues al igual que afirman otros autores y tal y como se refleja en la figura 3 , ha habido un cambio evolutivo en la terapéutica de descontaminación digestiva a favor del carbón activado ${ }^{23}$, pero bajo número de casos no permite establecer conclusiones definitivas, sino apuntar una tendencia. No obstante, dado que el intervalo asistencial aparece registrado en muy pocos casos, y que este dato es necesario para valorar la correcta indicación de la descontaminación digestiva, no podemos afirmar que exista una mejor indicación de estas técnicas. Lo que sí queda demostrado es la necesidad de mejorar este aspecto de la anamnesis toxicológica y su consignación en la historia clínica del paciente.

Este aspecto constituye una de las limitaciones de nuestro trabajo, al igual que el hecho de ser un estudio retrospectivo y unicéntrico, por lo que no es posible generalizar los resultados.

Si se comparan las cifras de ingreso hospitalario y mortalidad, resultan mayores con respecto a estudios de ámbito nacional con población general que se encuentra en un rango entre el 0 y el 0,1 $9 \% 1,16,19,21$, pero no distan mucho de la de mortalidad encontrada en otros estudios con población de edad avanzada de ámbito internacional $(0,86 \%-1,13 \%)^{10,28}$. Estos datos son congruentes con los tóxicos más implicados en el intento autolítico y en los fallecimientos, lo que confiere un perfil de mayor gravedad a la intoxicación ${ }^{38}$.

En conclusión, las intoxicaciones más frecuentes son las domésticas, con un mayor porcentaje de intoxicaciones por cáusticos y plaguicidas, lo cual conlleva una mayor necesidad de ingresos hospitalarios y mortalidad. Los resultados de nuestro trabajo deben confirmarse con otros estudios de tipo multicéntrico, aunque en cualquier caso creemos necesario que se extremen las medidas preventivas de aislamiento social, etc. para evitar las intoxicaciones en estos pacientes. 


\section{BIBLIOGRAFÍA}

1. GonzÁlez-Fernández D, Alonso-Fernández M. Intoxicaciones agudas en un Servicio de Urgencias. Estudio descriptivo en el área Sanitaria III de Asturias. Rev Toxicol 2009; 26: 122-127.

2. Caballero PJ, Dorado S, Díaz A, García Me, YuBERo L, Torres $\mathrm{N}$ et al. Vigilancia epidemiológica de la intoxicación aguda en el área sur de la Comunidad de Madrid: estudio VEIA 2004. An Med Interna (Madrid) 2008; 25: 262-268.

3. Doak MW, Nixon AC, Lupton DJ, Waring WS. Self-poisoning in older adults: patterns of drug ingestion and clinical outcomes. Age Ageing 2009; 38: 407-411.

4. Epelde F, Iglesias-Lepine ML, Anarte L. En plena crisis económica: coste y efectividad de las unidades de estancia corta hospitalarias. An Sist Sanit Navar 2012; 35: 469-475.

5. Casas-Herrero A, Izquierdo M. Ejercicio físico como intervención eficaz en el anciano frágil. An Sist Sanit Navar 2012; 35: 69-85.

6. De la Cruz-SÁnchez E, Torres-Bonete MD, García-Pallarés J, Gascón-Cánovas JJ, ValeroValenzuela A, Pereñíguez-Barranco JE. Dolor de espalda y limitación de la actividad física cotidiana en la población adulta española. An Sist Sanit Navar 2012; 35: 241-249.

7. Elvira Martínez CM, Fernández C, González Del Castillo J, González-Armengol JJ, Villarroel P, Martín-SÁnchez FJ. Modelo predictor de ingreso hospitalario a la llegada al servicio de urgencias. An Sist Sanit Navar 2012; 35: 207-217.

8. Chan J, Draper B, Banerjee S. Deliberate self-harm IN OLDER ADULTS: A REVIEW OF THE LITERATURE FROM 1995 to 2004. Int J Geriatr Psychiatry 2007; 22: 720-732.

9. HeISEL MJ. Suicide and its prevention among older adults. Can J Psychiatry 2006; 51: 143-154.

10. Cassidy N, LeE SKK, Donegan CF, Tracey JA. Poisoning in older adults: the experience of the National Poisons Information Centre. Ir Med J 2008; 101: 268-270.

11. Pérez de Ciriza JA, Pinillos MA, Aldaz J. Drogas emergentes y alimentos. An Sist Sanit Navar 2013; 36: 329-32.

12. Calderón Hernanz B, Oliver Noguera A, Tomás Vecina S, Baena Parejo Mi, García Peláez M, JuaNEs Borrego A et al. Conciliación de los medicamentos en los servicios de urgencias. Emergencias 2013; 25: 204-217.

13. Burillo-Putze G, Aldea-Perona A, Rodríguez-JiménEz C, García-SÁiz M, Climent-Díaz B, Munne-Mas $\mathrm{P}$ et al. Drogas Emergentes (II): El fenómeno del Pharming. An Sist Sanit Navar 2013; 36: 99-114.
14. Haselberger MB, Kroner BA. Drug poisoning in older patients. Preventative and management strategies. Drugs \& Aging 1995; 7: 292297.

15. BAtes N, Dines A. The risk of poisoning in later life. Elder Care 1999; 11: 8-11.

16. Carpintero JM, Ochoa FJ, Ruiz Ji, Bragado L, Palacios G, Ramalle-Gómara E et al. Prevalencia de las intoxicaciones agudas en Urgencias de La Rioja. Emergencias 2000; 12: 92-97.

17. Burillo G, Munne P, Dueñas A, Pinillos MA, NAVIERo JM, Сово J et al. National multicentre study of acute intoxication in emergency departments of Spain. Eur J Emerg Med 2003; 10: 101-104.

18. Pasto L, Martorell C, Mercadal G. Intoxicaciones agudas en el servicio de urgencias de un hospital universitario de nivel III: Cambios producidos en los últimos 10 años. Rev Toxicol 2006; 23: 95-100.

19. Amigo M, Nogué S, Gómez E, Sanjurjo E, SÁnchez M, Puiguriguer J. Medida de la calidad asistencial que se ofrece a los pacientes con intoxicaciones agudas en el servicio de urgencias. Emergencias 2006; 18: 7-16

20. Ferrer A, Nogué S, Dueñas A, Civeira E, Bajo A, Royo $\mathrm{R}$ et al. Perfil epidemiológico actual de las intoxicaciones agudas en urgencias. En: Net A, Marruecos L editores. Intoxicaciones agudas graves. Barcelona: Ars Medica; 2006: $1-15$.

21. Burillo G, Munne P, Dueñas A, Trujillo MM, JiméNEZ A, ADRIÁn MJ et al. Intoxicaciones agudas: perfil epidemiológico y clínico, y análisis de las técnicas de descontaminación digestiva utilizadas en los servicios de urgencias españoles en el año 2006. Emergencias 2008; 20: 1526

22. Perez P, Nogué S, Ríos J, Tejero I, Alonso JR. Evaluación de las intoxicaciones agudas por productos químicos atendidas en un Servicio de Urgencias. Med Clin (Barc) 2011; 136 : 149-152.

23. Burillo G, Mesa J. Toxicología clínica, urgencias y urgencias pediátricas. Emergencias 2012; 24: 346-347.

24. Puiguriguer J, Nogué S, Echarte Jl, Ferrer A, DueÑas A, García L et al. Mortalidad hospitalaria por intoxicación aguda en España (EXITOX 2012). Emergencias 2013; 25:467-471.

25. Cambridge DR, Wood RJ, Bateman DN. The epidemiology of self-poisoning in the UK. Br J Clin Pharmacol 2003; 56: 613-619.

26. Sesma J. Saturación en los servicios de urgencias hospitalarios. An Sist Sanit Navar 2012; 35: 195-198. 
27. Friedmann PD, Jin L, Karrison T, Nerney M, HaYLEY DC, MULLIKEN R et al. The effect of alcohol abuse on the health status of older adults seen in the emergency department. AM J Drug Alcohol Abuse 1999; 25: 529-542.

28. Mülberg W, Becher K, Heppner HJ, Wicklein S, SIEBER C. Acute poisoning in old and very old patients: a longitudinal retrospective study of 5883 patients in a toxicological intensive care unit. Z Gerontol Geriat 2005; 38: 182-189.

29. Pichot MH, Auzepy P, Richard C. Acute drug poisoning in suicidal elderly patients $70^{\circ} \mathrm{s}$ old and over. 92 cases in a medical ICU. Ann Med Interne 1990; 141: 429-430.

30. Alcalde P, Dapena MD, Nieto MD, Fontecha BJ. Ingreso hospitalario atribuible a efectos adversos a medicamentos. Rev Esp Geriatr Gerontol 2001; 36: 340-344.

31. Blasco F, Martinez J, Pérez R, Villares P, CarreÑo MC, Roman F. Estudio piloto sobre el consumo de fármacos en ancianos que ingresan en un hospital. An Med Intern 2004; 21: 69-71.

32. Suárez A, Pobes A, Quiñones L, Forascepi R. Los riesgos de la digoxina en el anciano. Nefrologia 2010; 30: 131-142.

33. Nogué S, Cino J, Civeira E, Puiguriguer J, Burillo $\mathrm{G}$, Dueñas A et al. Tratamiento de la intoxica- ción digitálica. Bases para el uso de los anticuerpos antidigital. Emergencias 2012; 24: 462-475.

34. Buitrago F. Métodos de medida de la adecuación del tratamiento farmacológico en pacientes pluripatológicos, ancianos o polimedicados. Aten Primaria 2013; 45: 19-20

35. Torres LF, Morales JM, Jiménez M, Copé G, SÁNchez S, Gómez JM. Impacto del autocuidado y manejo terapéutico en la utilización de los recursos sanitarios urgentes por pacientes crónicos: estudio de cohortes. Emergencias 2013; 25: 353-360.

36. American Academy of Clinical Toxicology, European Association of Poison Centers and Clinical Toxicologists. Position paper: single-dose activated charcoal. Clin Toxicol 2005; 43: 6187.

37. American academy of clinical toxicology, european association of poison centers and clinical toxicologists. Position paper: gastric lavage. Clin Toxicol 2004; 42: 933-943.

38. Ambrosio L, Pumar-Méndez MJ. Factores del entorno de trabajo que influyen en la ocurrencia de errores de administración de medicación. An Sist Sanit Navar 2013; 36: 77-85. 